\title{
Escenario de sepsis refractaria e insuficiencia suprarrenal, para promover razonamiento clínico en estudiantes de medicina
}

\author{
Scenario of refractory sepsis and adrenal insufficiency, \\ to promote clinical reasoning in medicine students \\ Cristian Labarca-Solar,*,‡ Simón Reyes-Morales, * Paz Valenzuela-Bodenburg, ${ }^{*}$ \\ Ignacio Tapia-Trucco, ${ }^{*, \ddagger}$ Soledad Armijo-Rivera*,‡
}

Palabras clave:

Simulación,

compromiso de conciencia, sepsis, sepsis refractaria,

insuficiencia

suprarrenal,

razonamiento clínico.

\section{Keywords: \\ Simulation, compromise of conscience, sepsis, refractory sepsis, adrenal insufficiency, clinical reasoning.}

\section{RESUMEN}

Introducción: El escenario de sepsis refractaria a tratamiento, por insuficiencia suprarrenal, permite enfrentar una situación que se presenta con un patrón típico y evoluciona de manera desfavorable pese a un manejo adecuado. Métodos: El escenario fue diseñado por un reumatólogo y un experto en simulación. La pauta de observación se basa en los criterios de manejo de neumonía adquirida en comunidad (NAC) y de sepsis. Resultados: El escenario fue utilizado en 12 simulaciones (86 estudiantes). El diagnóstico inicial de sepsis por NAC se estableció en $100 \%$ de los casos. La sospecha de una condición subyacente que pudiera explicar la falta de respuesta requirió del uso de salvavidas. Varios de los tratantes no llegaron a administrar corticoides. En el debriefing fue necesario reforzar el aprendizaje utilizando ayudas cognitivas sobre criterios SIRS, qSOFA, SOFA y Surviving Sepsis Campaign. Conclusiones: El escenario de simulación de sepsis refractaria a manejo inicial requiere una mirada integral del paciente, con conocimiento sobre la aproximación clínica al compromiso de conciencia y manejo inicial de shock séptico. Adicionalmente, es posible la evaluación del razonamiento clínico inicial y las estrategias cognitivas analíticas utilizadas para volver a abrir el espectro diagnóstico cuando las cosas no suceden como se espera.

\section{ABSTRACT}

Introduction: The scenario of sepsis refractory to treatment, due to adrenal insufficiency, allows us to face a situation that presents a typical pattern and evolves unfavorably despite proper management. Methods: The scenario was designed by a rheumatologist and a simulation expert. The observation guideline is based on the criteria of management of community-acquired pneumonia and sepsis. Results: The scenario was used in 12 simulations (86 students). The initial diagnosis of sepsis by communityacquired pneumonia was established in $100 \%$ of the cases. Suspicion of an underlying condition that could explain the lack of response required the use of scenario lifesavers. Several of the students failed to administer corticosteroids. In the debriefing it was necessary to reinforce the learning using cognitive aids on SIRS criteria, qSOFA, SOFA and Surviving Sepsis Campaign. Conclusions: The simulation scenario of sepsis refractory to initial management requires a comprehensive look at the patient, with knowledge about the clinical approach to the compromise of conscience and initial management of septic shock. Additionally, it is possible to evaluate the initial clinical reasoning and the cognitive strategies used to broad the diagnostic spectrum when things do not happen as expected.

\section{INTRODUCCIÓN}

* Escuela de Medicina, Facultad de Medicina Clínica Alemana Universidad del Desarrollo. ‡ Centro de Simulación y Seguridad Clínica, Facultad de Medicina Clínica Alemana Universidad del Desarrollo.
$\mathrm{R}_{\mathrm{t}}^{\mathrm{z}}$ acionalidad educacional del escenario: entendiendo la importancia de la sepsis como causa de morbimortalidad y costos elevados a nivel mundial ${ }^{1-4}$ y en Chile, ${ }^{5}$ resulta imprescindible que la identificación y el manejo oportuno sea una competencia transversal a todas las escuelas de medicina.

En un intento por combatir este problema de salud pública, diversas instituciones internacionales han desarrollado guías para la orientación del equipo de salud desde la sospecha hasta el manejo específi- co de la sepsis. ${ }^{6,7}$ Sin embargo, es relevante generar un espacio de reflexión sobre la aplicabilidad de estas guías en la epidemiología local, comprendiendo que los score clínicos (SIRS/SOFA) son un apoyo pero no una receta infalible para todos los pacientes. ${ }^{8}$

En relación al proceso de razonamiento clínico, en los escenarios de simulación de urgencia los estudiantes hacen visibles los procesos de recogida, integración o confirmación de la información. ${ }^{9}$ Asimismo, es posible interpretar durante el escenario y reconocer durante los debriefing, cuál de los sistemas definidos en la teoría de procesa- 
miento dual es el que está guiando las decisiones en los diferentes momentos de la simulación. ${ }^{10}$

Además es posible analizar los elementos cognitivos y no cognitivos que influencian las acciones de los estudiantes, generando errores en la toma de decisiones. ${ }^{10,11}$ Por otro lado, entender las brechas cognitivas a las que se enfrentan los estudiantes cuando las intervenciones teóricamente correctas no tienen el resultado esperado es otra dimensión susceptible de analizar. ${ }^{11}$

\section{PRESENTACIÓN DEL CASO}

Información del escenario

Título: sepsis refractaria e insuficiencia suprarrenal.

Nombre del paciente: Carlos Jofré Jofré.

Desarrolladores: Cristian Labarca, Soledad Armijo.

Fecha de diseño: marzo 2019.

Apropiado para: estudiantes e internos de medicina, residentes de medicina y urgencia, médicos y enfermeras de urgencia y cuidado crítico.

\section{Diseño del escenario}

El escenario fue diseñado por un reumatólogo y un experto en simulación, para ser implementado en un simulador de alta fidelidad, y fue validado por un segundo internista.

El escenario inicia con un paciente con compromiso cualitativo de conciencia, incapaz de entregar información. El paciente no porta identificadores que indiquen su condición de usuario crónico de corticoides. Se presenta acompañado con un familiar, que actúa como confederado. El familiar no vive con el paciente, al momento de la llegada a la urgencia se encuentra nervioso y relata los síntomas respiratorios característicos de una neumonía, entregando información parcial sobre condiciones previas y medicamentos.

El algoritmo de programación contempla inestabilidad hemodinámica progresiva pese a las acciones que realicen los participantes, y sólo mejora cuando se identifica la necesidad de administrar corticoides exógenos (Figura 1). No existe ficha electrónica que permita ver de inmediato los antecedentes del paciente.

Los criterios de observación se basaron en los criterios clínicos de manejo de neumonía adquirida en comunidad (Tabla 1),12 criterios qSOFA y SOFA ${ }^{7}$ y recomendaciones Surviving Sepsis Campaign ${ }^{6}$ para el manejo en las primeras tres horas del paciente séptico.

\section{Objetivos}

- Demostrar una evaluación primaria del paciente.

- Demostrar un enfrentamiento inicial de compromiso de conciencia adecuado.

- Identificar signos de sepsis y shock séptico.

- Realizar manejo básico de reanimación adecuado al paciente.

- Realizar examen físico dirigido a identificar foco de infección.

- Solicitar exámenes para identificar la etiología precozmente.

- Iniciar el manejo empírico precoz para sepsis de foco respiratorio.

- Reconocer la refractariedad al tratamiento y buscar causas subyacentes.

- Reconocer la insuficiencia suprarrenal secundaria relativa.

- Reconocer la necesidad de traslado a una Unidad de Paciente Crítico.

- Reconocer errores de fijación y establecer estrategias de corrección adecuadas.

\section{Acciones críticas}

- Reconocer sepsis usando criterios qSOFA, sin retrasar diagnóstico.

- Reponer volumen de manera enérgica en las primeras tres horas.

- Tomar hemocultivos previo al inicio de antibiótico empírico correcto en NAC.

- Reconocer necesidad de uso de corticoides.

\section{Preparación del escenario}

- Box servicio de urgencias.

- Monitor de signos vitales.

- Simulador de alta fidelidad adulto.

- Hemoglucotest.

- Carro de paro equipado con mascarilla venturi-mascarilla de alto flujo, 2 vías venosas periféricas (VVP) \#18 y 16, bolsa mascarilla, oxígeno, sueros y drogas vasoactivas.

- Radiografía de tórax con neumonía extensa.

\section{Recomendaciones para la conducción del escenario}

El escenario fue diseñado para que un estudiante actúe como familiar del paciente asumiendo el rol de confederado, dos estudiantes participan como enfermero y como técnico de enfermería experimentados, y otros dos asumen el rol de hot seat de manera secuencial, uno actuando como el médico 


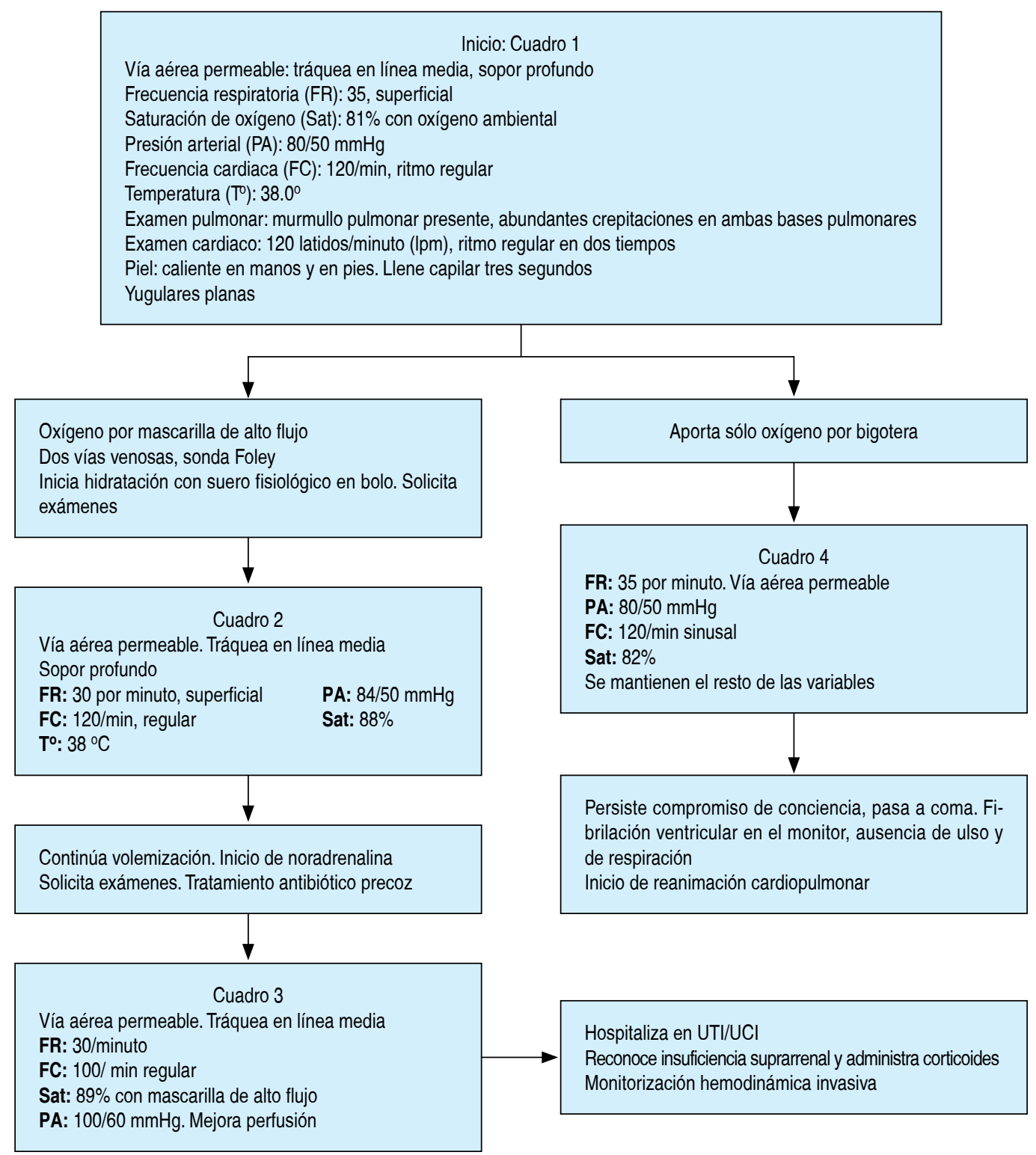

Figura 1:

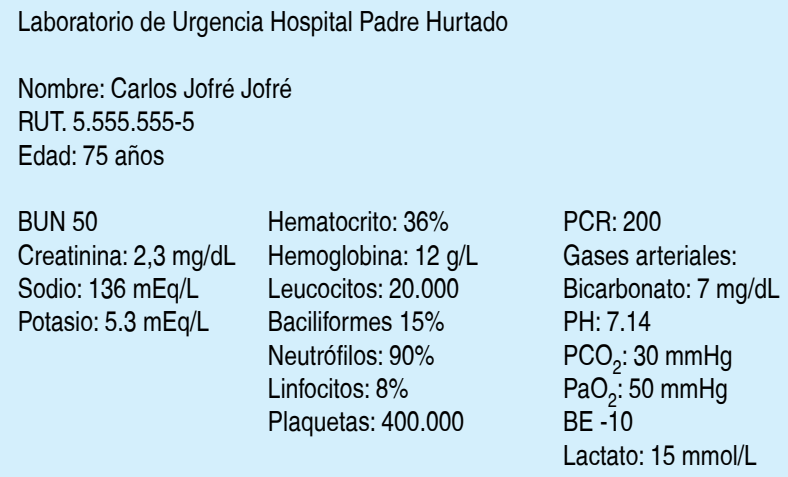

Algoritmo de manejo del escenario $y$ exámenes del paciente. 
a cargo de la primera atención y el segundo actuando como el médico que recibe el turno y al paciente para continuar su atención. Este último estudiante se integra al escenario cuando lo indica el instructor, una vez que el primer médico tiene ya decidido un primer diagnóstico y abordaje terapéutico.

Instrucciones al confederado (se entregan en privado) usted acompaña a su padre, Carlos Jofré de 75 años. No vive con él. Su padre padece de hipertensión arterial y artritis reumatoide en tratamiento inmunosupresor con prednisona y metotrexato. Desde hace tres días su padre inicia cuadro caracterizado por tos, expectoración mucopurulenta, fiebre y posteriormente compromiso de conciencia progresivo. No entregue más información al comienzo, actúe con nerviosismo.

Sólo si le preguntan de manera directa indique los fármacos de uso diario:

- Amlodipino 10 mg/día.

- Losartán 100 mg/día.

\section{Tabla 1: Instrumento de evaluación.}

\begin{tabular}{l}
$\begin{array}{l}\text { Pauta de evaluación competencias sepsis de } \\
\text { foco pulmonar }\end{array}$ \\
\hline
\end{tabular}

Evalúa cumplimiento de medidas de precaución universal en equipo de trabajo

Solicita monitorización signos vitales

Monitorización electrocardiograma continua

Solicita Hemoglucotest

Solicita electrocardiograma

Evalúa permeabilidad de vía aérea

Aporta $\mathrm{O}_{2}$ por mascarilla de alto flujo

Instala dos vías venosas periféricas gruesas

Instalación de sonda Foley

Indica volemización precoz

Indica tratamiento antibiótico de amplio espectro

Solicita exámenes generales

Solicita radiografía de tórax portátil

Interpreta de manera adecuada exámenes generales

Indica uso de drogas vasoactivas (noradrenalina)

Indica intubación y ventilación mecánica

Indica hospitalización en Unidad de Cuidados

Intensivos

Solicita monitorización invasiva

Reconoce insuficiencia suprarrenal como posible

causa de falta de respuesta

Indica corticoides

Entrega adecuadamente al paciente a médico

residente de UPC

Nota
- Furosemida 40 mg/día.

- Metotrexato 20 mg una vez a la semana.

- Prednisona 10 mg/día.

\section{Preguntas para orientar el debriefing}

- ¿Por qué este cuadro es una urgencia que requiere tratamiento oportuno?

- ¿Cuál es el manejo de reanimación inicial de un paciente séptico?

- ¿Cuál es la importancia del tratamiento antibiótico precoz en la sepsis?

- ¿Cuáles son los riesgos de una infección en un paciente inmunosuprimido?

- ¿Cuáles son los efectos a nivel suprarrenal del uso crónico de corticoides?

- ¿En qué unidad trata usted a su paciente?

\section{DISCUSIÓN}

\section{Resultados de la implementación:}

El escenario fue utilizado en 12 simulaciones (86 estudiantes). El diagnóstico inicial de sepsis por NAC se estableció en $100 \%$ de los casos. La sospecha de una condición subyacente que pudiera explicar la falta de respuesta requirió del uso de salvavidas instalados por los instructores durante el escenario. Varios de los tratantes no llegaron a administrar corticoides de manera espontánea durante el escenario, y requirieron que alguno de los miembros del equipo hiciera la sugerencia. En el debriefing fue necesario reforzar el aprendizaje utilizando ayudas cognitivas sobre criterios SIRS, qSOFA, SOFA y Surviving Sepsis Campaign y sobre las causas de insuficiencia suprarrenal secundaria, particularmente asociada a corticoides.

\section{Discusión sobre el proceso y la utilidad del escenario}

Diseñar un escenario con una presentación clínica inicial típica y muy clara, pretende llevar a los participantes a tomar decisiones usando el sistema intuitivo, una forma rápida de pensamiento. ${ }^{13}$ La ausencia de la respuesta esperada frente a un tratamiento adecuado debiera llevar al uso de un pensamiento más analítico, profundo y lento, útil para minimizar el error. ${ }^{13}$

En esta línea, y sobre todo en sepsis, la deliberación durante el escenario y la discusión en el debriefing de los diagnósticos diferenciales y factores concomitantes que influyen en la mortalidad y la falla a tratamiento, abren un espacio para el abordaje de la insuficiencia suprarrenal relativa ${ }^{14}$ 
como brecha cognitiva en un ambiente seguro y de aprendizaje para practicar el razonamiento clínico mediante uso alternativo de estrategias de pensamiento intuitivo y analítico. ${ }^{13}$

\section{CONCLUSIONES}

El escenario de simulación de sepsis refractaria a manejo inicial requiere una mirada integral del paciente, con conocimiento sobre la aproximación clínica al compromiso de conciencia y manejo inicial de shock séptico, y a la causa específica del cuadro, en este caso, la neumonía adquirida en la comunidad. Es un caso adecuado para lograr la integración de contenidos y la demostración de competencias antes del ingreso al internado, en estudiantes de medicina.

Adicionalmente, es posible la evaluación del razonamiento clínico inicial y las estrategias cognitivas analíticas utilizadas para volver a abrir el espectro diagnóstico cuando las cosas no suceden como se espera.

El adecuado manejo del escenario requiere que los instructores estén familiarizados con la metodología de simulación, tanto en el briefing como en la conducción de escenarios, utilizando salvavidas que no perturben el realismo y permitan mantener los objetivos de la simulación, y en este caso en particular, que manejen los conceptos de razonamiento clínico, las etapas de este proceso, y los tipos de errores cognitivos y estrategias para enfrentarlos que pueden ser utilizados durante los debriefing.

\section{AGRADECIMIENTOS}

El equipo agradece la gentileza del Dr. Osvaldo Llanos Valdés, Médico Internista y Especialista en Cuidado Crítico, Subdirector Médico de Clínica Bicentenario, por su colaboración como validador independiente del escenario y por su especial contribución a identificar las acciones críticas identificadas en el mismo.

\section{REFERENCIAS}

1. Angus DC, Linde-Zwirble WT. Epidemiology of severe sepsis in the United States: analysis of incidence, outcome, and associated costs of care. Crit Care Med. 2001; 29 (7): 1303-1310. https://www.ncbi.nlm.nih. gov/pubmed/11445675.

2. Hatfield KM, Dantes RB. Assessing variability in hospitallevel mortality among US medicare beneficiaries with hospitalizations for severe sepsis and septic shock. Crit Care Med. 2018; 46 (11): 1753-1760. https://journals. Iww.com/ccmjournal/Citation/2018/11000/Assessing Variability_in_Hospital_Level_Mortality.6.aspx
3. Rhee C, Dantes R. Incidence and trends of sepsis in US Hospitals Using Clinical vs Claims Data. JAMA. 2017; 318 (13): 2009-2014. Sitio web: https://www. ncbi.nlm.nih.gov/pubmed/28903154.

4. Raymund B, Dantes LE. Combatting sepsis: a public health perspective. Clin Infect Dis. 2018; 67 (8): 1300-1302. Sitio web: https://academic.oup.com/cid/ article/67/8/1300/5019029

5. Dougnac LA, Mercado FM, Cornejo RR, Cariaga VM, Hernández PG, Andresen HM, et al. Prevalencia de sepsis grave en las Unidades de Cuidado Intensivo: primer estudio nacional multicéntrico. Revista Médica de Chile. 2007; 135(5): 620-630. https://dx.doi. org/10.4067/S0034-98872007000500010.

6. Rhodes A, Evans LE, Alhazzani W, Levy MM, Antonelli M, Ferrer R, et al. Surviving Sepsis Campaign: International Guidelines for Management of Sepsis and Septic Shock: 2016. Intensive Care Med. 2017; 43 (3): 304-377. doi: 10.1007/s00134-017-4683-6. Epub 2017 Jan 18.

7. Singer $M$, Deutschmann CS, Seymour CW, ShankarHari M, Annane D, Bauer M, et al. The Third International Consensus Definitions for Sepsis and Septic Shock (Sepsis-3). JAMA. 2016; 315 (8): 801810. doi: 10.1001/jama.2016.0287.

8. Romero C, Luengo C. Recomendaciones SOCHIMI para el manejo inicial de la sepsis. Revista Chilena de Medicina Intensiva. 2017; 32 (2): 107-126. Sitio web: https://www.medicina-intensiva.cl/docs/ Recomendaciones_SOCHIMI_Sepsis._Final.pdf.

9. Audétat MC, Laurin S, Dory V, Charlin B, Nendaz MR. Diagnosis and management of clinical reasoning difficulties: Part I. Clinical reasoning supervision and educational diagnosis. Med Teach. 2017; 39 (8): 792-796. doi: 10.1080/0142159X.2017.1331033. Epub 2017 Jun 7.

10. Audétat MC, Laurin S, Dory V, Charlin B, Nendaz MR. Diagnosis and management of clinical reasoning difficulties: Part II. Clinical reasoning difficulties: management and remediation strategies. Med Teach. 2017; 39 (8): 797-801. doi: 10.1080/0142159X.2017.1331034. Epub 2017 Jun 7.

11. Stiegler MP, Neelankavil JP, Canales C, Dhillon A. Cognitive errors detected in anaesthesiology: a literature review and pilot study. Br J Anaesth. 2012; 108 (2): 229-235. doi: 10.1093/bja/aer387. Epub 2011 Dec 8.

12. Saldías F, Pérez C. Manejo de la neumonía del adulto adquirida en la comunidad. Resumen del Consenso Nacional. Rev Med Chile. 2005; 133: 953-967.

13. Croskerry P, Petrie DA, Reilly JB, Tait G. Deciding about fast and slow decisions. Acad Med. 2014; 89 (2): 197200. doi: 10.1097/ACM.0000000000000121.

14. Casares VM, Raurich PJ. Septic shock and relative adrenal insufficiency. Med Intensiva. 2003; 27 (8): 525-530. Sitio web: http://www.medintensiva. org/es-shock-septico-e-insuficiencia-suprarrenalarticulo-13053470.

Correspondencia:

Soledad Armijo Rivera

Avenida Las Condes Núm. 12438,

Lo Barnechea, Santiago, Chile,

Tel: + 56996796889

E-mail: s.armijo@udd.cl

soledad.armijo@gmail.com 